\title{
Molekularbiologischer Nachweis von Tumormarkern im peripheren Blut und Plasma/Serum von Patienten mit malignem Melanom
}

\author{
G. Rappl \\ S. Ugurel \\ W. Tilgen \\ U. Reinhold
}

\author{
Molecular Detection of Tumor Markers in Peripheral Blood and Plasma/Serum \\ of Patients with Malignant Melanoma
}

\section{Zusammenfassung}

Der frühe Nachweis einer Metastasierung beim malignen Melanom ist von entscheidender Bedeutung für die Prognose der Erkrankung. Zum Nachweis zirkulierender Tumorzellen wurden im Laufe der Jahre zunehmend sensitivere Methoden eingesetzt. Durch die Entwicklung der revers-transkribierten Polymerasekettenreaktion (RT-PCR) wurde ein spezifischer und sensitiver Nachweis zirkulierender Tumorzellen möglich. Beim malignen Melanom wurden mittels RT-PCR melanomspezifische Transkripte, insbesondere die Expression des Genes für das Enzym Tyrosinase in umfangreichen Studien untersucht. Weiterführende Projekte untersuchten die Expression weiterer Melanom-assoziierter Transkripte („Multimarker RT-PCR“), sowie den Einsatz der quantitativen „real-time“-RT-PCR zur Optimierung der Sensitivität und Spezifität zur Detektion von Mikrometastasen. Einen weiteren noch in der experimentellen Phase befindlichen Ansatz zum Nachweis von Mikrometastasen stellt die Analyse von frei zirkulierenden tumorassoziierten Nukleinsäuren im Plasma/Serum dar. Erste Daten bestätigen die prinzipielle Möglichkeit dieser Nachweismethodik als neuen Ansatz für den Nachweis einer Mikrometastasierung.

\section{Abstract}

The early detection of metastasis in malignant melanoma is important for prognosis of disease. For detection of circulating tumor cells more sensitive methods were developed during past years. Through the development of polymerase-chain-reaction a specific and sensitive detection of circulating tumor cells is possible. The expression of melanoma-associated transcripts in malignant melanoma, especially the expression of the tyrosinase gene, was analyzed in multiple studies. More recent studies analyze expression of different melanoma-associated transcripts (multi-marker RT-PCR) or usage of real-time RT-PCR for optimization of detection sensitivity and specificity of micrometastases. A new method under development is the detection of tumorassociated nucleic acids in plasma/serum. First data confirm the possible usage of this method for detection of micrometastases.

\section{Begünstigende Faktoren für eine Mikrometastasierung}

Die Tumorausbreitung stellt beim malignen Melanom den entscheidenden und Prognose bestimmenden Faktor dar. $\mathrm{Zu}$ den wichtigsten klinischen Parametern, mit denen sich die Tumorausbreitung abschätzen lässt, zählen die maximale Dicke des Primärtumors und eine mögliche Beteiligung der regionären Lymphknoten. Beim malignen Melanom hat sich die vertikale
Tumordicke nach Breslow als wichtigster prognostischer Parameter in Primärstadien erwiesen [1]. Für die weiteren Tumorstadien wird als wichtiger Parameter die Beteiligung der Lymphknoten berücksichtigt. Tumorlokalisation und Geschlecht des Patienten stellen einen weiteren Prognoseparameter dar [1]. Im Stadium der Metastasierung werden als unabhängige Prognoseparameter eine LDH-Erhöhung und eine Albuminerniedrigung $[2,3]$ beschrieben. Die Gesamtzahl dieser Faktoren macht deut-

Institutsangaben

Universitäts-Hautklinik und Poliklinik, Universitätskliniken des Saarlandes,

Homburg/Saar

Korrespondenzadresse

Prof. Dr. med. Uwe Reinhold · Universitäts-Hautklinik und Poliklinik · Universitätskliniken des Saarlandes · 66421 Homburg/Saar·E-mail: uwe.reinhold@uniklinik-saarland.de 
lich, dass eine stattgefundene Metastasierung des Primärtumors und deren Etablierung bzw. Progression für die Prognose des Krankheitsverlaufes den entscheidenden Faktor darstellt. Ein frühzeitiger Nachweis einer beginnenden Metastasierung ist daher für die Diagnostik und eventuell Therapieeinleitung von großer Bedeutung.

\section{Nachweis zirkulierender Tumorzellen mittels Lichtmikroskopie und Immunhistochemie}

Als erste Nachweistechnik zirkulierender Tumorzellen diente die Lichtmikroskopie. In Blutausstrichen wurde versucht, Tumorzellen anhand ihrer Morphologie zu identifizieren. Die Sensitivität dieser Technik war jedoch gering (s. Tab.1). Mit der Einführung von immunhistochemischen Nachweismethoden konnte die Sensitivität verbessert werden (s. Tab.1). Eine Bedeutung in der Routinediagnostik zum „Staging“ von Krebspatienten hat die Methodik jedoch nicht erlangt [4]. Falsch negative Resultate waren insbesondere durch den Verlust melanomspezifischer Antigene sowie Antikörperkreuzreaktionen etc. bedingt $[5,6]$.

\section{Nachweis von Melanommikrometastasen mittels Polymerasekettenreaktion}

Ein verbesserter Nachweis einer Mikrometastasierung wurde durch den Einsatz der Polymerasekettenreaktion möglich. Diese Technik erlaubt im Vergleich zu immunhistochemischen Nachweismethoden einen spezifischeren und sensitiveren Nachweis zirkulierender Tumorzellen (s. Tab.1). Das Verfahren wurde kurz nach der eigentlichen Entwicklung im Jahre 1987 zum Nachweis zirkulierender Tumorzellen von Melanompatienten eingesetzt. Smith et al. [7] wiesen 1991 im peripheren Blut bei drei von fünf Patienten mRNA für das melanomassoziierte Antigen Tyrosinase nach. Brossart et al. [8] fanden im peripheren Blut bei $100 \%$ der untersuchten Patienten im Tumorstadium IV und bei 35\% der Patienten im Stadium III im peripheren Blut Tyrosinase mRNA. In den darauf folgenden Jahren wurden zahlreiche Studien zum Nachweis von Tyrosinase mRNA im peripheren Blut von Melanompatienten publiziert (s. Tab. 2). Die Resultate waren jedoch äußerst divergent und die Anzahl der TyrosinasemRNA-positiven Patienten variierte sehr stark (s. Tab. 2). Im Tumorstadium IV waren in einigen Studien 0\%, in anderen Studien $100 \%$ der Patienten positiv für Tyrosinase mRNA. Im Durchschnitt waren im Stadium IV 20-30\% , in den Stadien I-III 0-15\% der Patienten positiv für den Tumormarker Tyrosinase. Eine Ursache für die unterschiedlichen Ergebnisse bestand möglicherweise in unterschiedlichen methodischen Details des Nachweisverfahrens. So wurden verschiedene Techniken zur Isolation von

Tab. 1 Nachweisgrenze zirkulierender Tumorzellen im peripheren Blut

\begin{tabular}{ll} 
Technik & Nachweisgrenze \\
\hline Lichtmikroskopie & 1 Tumorzelle in $10^{2}-10^{3}$ Blutzellen \\
\hline Immunhistochemie & 1 Tumorzelle in $10^{4}-10^{5}$ Blutzellen \\
\hline PCR & 1 Tumorzelle in $10^{6}-10^{8}$ Blutzellen \\
\hline
\end{tabular}

RNA bzw. mRNA aus dem peripheren Blut, sowie unterschiedliche PCR-Protokolle zur Amplifikation der Transkripte eingesetzt. Daher wurde zur Qualitätssicherung 1997 von der Europäischen Organisation zur Erforschung und Behandlung von Krebserkrankungen (EORTC) ein Ringversuch in neun verschiedenen Laboratorien in Europa durchgeführt. Als Ergebnis wurde festgestellt, dass die Heterogenität beim Nachweis von Tyrosinase mRNA hauptsächlich in der Probenvorbereitung, der RNA-Extraktion und cDNA-Synthese begründet ist und weniger durch unterschiedliche PCR-Protokolle bedingt ist [9]. Mit der späteren Verfügbarkeit eines kommerziellen Kit zum Nachweis von Tyrosinase mRNA war ein standardisiertes Verfahren zur besseren Vergleichbarkeit der Ergebnisse der einzelnen Zentren verfügbar. Es wurde daher in Deutschland ein Ringversuch der Arbeitsgemeinschaft Dermatologische Onkologie (ADO) in sieben verschiedenen deutschen Zentren durchgeführt, der ein standardisiertes PCR-Verfahren zum Nachweis von Tyrosinase auf seine Sensitivität, Spezifität und Reproduzierbarkeit und damit Eignung für die Routinediagnostik von Mikrometastasen evaluieren sollte. Von allen teilnehmenden Laboren wurde übereinstimmend eine hohe Sensitivität und Spezifität der standardisierten Methodik bestätigt. Allerdings zeigen die Analysen von identisch aliquotierten Blutproben von Melanompatienten eine äußerst geringe Übereinstimmung der Ergebnisse, so dass der Nachweis von zirkulierenden Melanomzellen mittels PCR weiter als experimentell angesehen werden muss und derzeit für die Routinediagnostik nicht geeignet ist [10].

\section{Einsatz von Multimarker RT-PCR und „Real-time“ RT-PCR für den Nachweis einer Mikrometastasierung}

\section{Multimarker RT-PCR}

Eine weitere mögliche Ursache für die Diskrepanz der Ergebnisse zwischen den zahlreichen Studien zum Nachweis von Tyrosinase mRNA (s. Tab. 2) ist der häufige Verlust der Expression von melanomassoziierten Antigenen in Tumorzellen [11]. 1995 setzten Hoon et al. [12] eine PCR-Technik, die so genannte MultimarkerPCR zum Nachweis von Mikrometastasierung ein. Der Vorteil dieser Methode besteht darin, dass die Expression mehrerer melanomassoziierter Transkripte gleichzeitig analysiert wird, und somit auch Tumorzellen nachweisbar sind, die einen partiellen Verlust von Tumorantigenen aufweisen. In einer ersten Studie untersuchten Hoon et al. [12] an Melanomzellinien neben der Expression des Tyrosinase-Genes die Expression weiterer melanomassoziierter Antigene wie P97, MAGE-3 und MUC-18. In dieser Untersuchung wurde bei $80 \%$ der Melanomzelllinien eine mRNA-Expression aller vier untersuchten melanomassoziierten Antigene beobachtet. Die Spezifität dieser Methodik wurde dadurch bestätigt, dass in nur 2 von 39 Blutproben gesunder Spender Transkripte für MUC-18 nachweisbar waren. In einer anderen Untersuchung [13] wurde die Expression von mRNA für insgesamt fünf Gene der Melaninbiosynthese (Tyrosinase, TRP-1, TRP-2, Pmel 17/gp100 und MART-1/MelanA) analysiert [13]. Mittels dieser Multimarker-PCR wurden bei $47 \%$ der Patienten im Stadium I - IV alle fünf Tumorantigene und bei $86 \%$ der Patienten im Stadium I-IV mindestens ein Tumorantigen im Vollblut detektiert. In einem Kontrollkollektiv war in keinem der untersuchten Fälle einer der Tumormarker nachweisbar [14]. Von den Tumorantigenen wurde mit 86\% am häufigsten Tyrosinase im Blut von Patienten mit metastasiertem Melanom nachgewie- 
Tab. 2 Studien zum Nachweis von Tyrosinase mRNA im peripheren Blut von Melanompatienten*

\begin{tabular}{|c|c|c|c|c|c|c|c|}
\hline & $\begin{array}{l}\text { Gesamtzahl } \\
\text { Patienten }\end{array}$ & Stad. I & Stad. II & Stad. I/II & Stad. III & Stad. IV & $\begin{array}{l}\text { Negativ- } \\
\text { kontrollen }\end{array}$ \\
\hline Smith 1991 & 7 & 0 & 0 & 0 & $0 / 1$ & $4 / 6$ & $0 / 8$ \\
\hline Battayani 1995 & 60 & NS & NS & $2 / 10$ & $8 / 18$ & $16 / 32$ & $0 / 14$ \\
\hline Hoon 1995 & 119 & 0 & 0 & $13 / 17$ & $31 / 36$ & $63 / 66$ & $0 / 39$ \\
\hline Foss 1995 & 6 & 0 & 0 & 0 & 0 & $0 / 6$ & $2 / 31$ \\
\hline Mellado 1996 & 91 & $4 / 17$ & $10 / 22$ & $14 / 39$ & $7 / 17$ & $33 / 35$ & $0 / 50$ \\
\hline Stevens 1996 & 12 & $0 / 12$ & $1 / 3$ & $1 / 5$ & $2 / 4$ & $2 / 3$ & $0 / 25$ \\
\hline Glaser 1997 & 102 & NS & NS & $1 / 43$ & $0 / 15$ & $12 / 44$ & $0 / 35$ \\
\hline Reinhold 1997 & 65 & NS & NS & $0 / 31$ & $1 / 21$ & $5 / 13$ & $0 / 20$ \\
\hline Jung 1997 & 50 & 0 & 0 & 0 & 0 & $13 / 50$ & $0 / 15$ \\
\hline Tessier 1997 & 85 & 0 & 0 & $0 / 42$ & $0 / 20^{\mathrm{a}}$ & $16 / 23$ & $0 / 20$ \\
\hline Voit 1999 & 64 & $0 / 18$ & $2 / 10$ & $2 / 28$ & $11 / 24$ & 9/12 & $0 / 15$ \\
\hline Palmieri 1999 & 235 & $27 / 87$ & $26 / 67$ & $53 / 154$ & $24 / 49$ & $24 / 32$ & $0 / 41$ \\
\hline Mellado 1999 & 57 & NS & NS & $2 / 11$ & $6 / 33$ & $2 / 13$ & $0 / 8$ \\
\hline Le Bricon 1999 & 30 & 0 & 0 & 0 & $1 / 10$ & $4 / 20$ & $0 / 1$ \\
\hline Schittek 1999 & 225 & $13 / 74$ & $8 / 45$ & $21 / 119$ & $8 / 48$ & $21 / 58$ & $0 / 40$ \\
\hline Curry 1999 & 186 & $4 / 13$ & $30 / 76$ & $34 / 89$ & $55 / 97$ & 0 & $0 / 50$ \\
\hline Hanekom 1999 & 165 & $4 / 76$ & $6 / 67$ & $10 / 143$ & $0 / 10$ & $0 / 12$ & $0 / 1$ \\
\hline Alao 1999 & 21 & 0 & 0 & 0 & $1 / 4$ & $5 / 17$ & $0 / 12$ \\
\hline Kopreski 1999 & 6 & 0 & 0 & 0 & 0 & $4 / 6$ & $0 / 20$ \\
\hline de Vries 1999 & 106 & 0 & 0 & $2 / 6$ & $4 / 27$ & $15 / 73$ & $0 / 10$ \\
\hline Proebstle 2000 & 212 & $12 / 106$ & $10 / 56$ & $22 / 162$ & $8 / 26$ & $16 / 24$ & 0 \\
\hline
\end{tabular}

* Die Daten zeigen die Anzahl Tyrosinase-positiver Patienten/Gesamtanzahl der Patienten des jeweiligen Tumorstadiums. NS: nicht separiert. a Proben waren bei der ersten Testung negativ. Patienten wurden ein zweites Mal getestet und waren bei einer Testung positiv für Tyrosinase mRNA.

sen. Zusammenfassend lässt sich feststellen, dass mit Hilfe der Multimarker-PCR im Vergleich zur Detektion nur eines Markers der Nachweis von zirkulierenden Melanomzellen in einem hohen Prozentsatz gelingt. Zukünftige Studien müssen untersuchen, ob und inwieweit dies erweiterte Verfahren für die Standarddiagnostik des malignen Melanoms geeignet ist.

\section{„Real-time“-RT-PCR}

Ein Nachteil der qualitativen RT-PCR-Techniken ist, dass die Konzentration von tumorassoziierten Transkripten nicht quantitativ gemessen werden kann. Über die Entwicklung der „RealTime“-RT-PCR-Technik wurde es jedoch möglich, die Schritte der Amplifikation, Detektion und Quantifizierung von Transkripten bzw. PCR-Produkten zu kombinieren. Die Technik zeichnet sich durch eine einfache Handhabung und kurze Analysezeiten aus. Weiterhin sind die einzelnen Amplifikationsreaktionen gut reproduzierbar und für einen hohen Probendurchsatz standardisierbar. Der Einsatz der „real-time“-RT-PCR zum Nachweis einer Mikrometastasierung bietet besonders Vorteile gegenüber den bisherigen RT-PCR-Techniken. Zum einen sind Aussagen zur Anzahl der in der Probe vorhandenen Transkripte möglich, zum anderen erhält man Informationen über die Qualität der isolierten
RNA. De Vries et al. [15] setzten erstmals eine „Real-time“-RTPCR in Kombination mit Multimarker-PCR für den Nachweis von Tumormarkern im peripheren Blut von Melanompatienten ein. Sie fanden heraus, dass die geringe Reproduzierbarkeit der Detektion zirkulierender Melanomzellen nicht auf Unterschiede in der mRNA-Qualität zurückzuführen ist, sondern durch eine geringe Anzahl von amplifizierbaren Tumortranskripten bedingt ist. In einer weiteren Studie wurden im Detail die Spezifität und Sensitivität der „Real-time“-RT-PCR zum Nachweis disseminierter Melanomzellen untersucht [16]. Als Ergebnis konnte mittels „nested“-RT-PCR 1 Tumorzelle in $1 \mathrm{ml}$ Vollblut spezifisch detektiert werden. Das RT-PCR-Protokoll zeichnete sich durch eine hohe Sensitivität, Effizienz, Spezifität und Reproduzierbarkeit aus. Die hohe Spezifität dieser Methode wird durch den Einsatz von genspezifischen Sonden zum Nachweis von Amplifikationsprodukten, Schmelzkurvenanalysen und anschließender Agarosegelelektrophorese der PCR-Produkte möglich. 
Nachweis tumorassoziierter zirkulierender mRNA im Plasma/Serum von Melanompatienten

Neue Perspektiven zum Nachweis einer Mikrometastasierung ergeben sich aus der erstmals 1998 beschriebenen Beobachtung, dass bei Patienten mit malignem Melanom tumorassoziierte extrazelluläre RNA frei im Serum zirkuliert [17]. In eigenen Studien konnten diese ersten Beobachtungen bestätigt werden [18]. Neuere Daten können diese Befunde auch bei anderen Tumorentitäten bestätigen (Übersicht in: Reinhold \& Schröder) [19]. Es gibt erste Hinweise darauf, dass zirkulierende extrazelluläre RNA im Serum/Plasma in fragmentierter Form (Größe bis ca. $400 \mathrm{bp}$ ) vorliegt (M. Fleischhacker et al. pers. Mitteilung). Die Existenz freier mRNA im Serum/Plasma wurde zunächst infrage gestellt, da im Serum/Plasma hohe Konzentrationen RNA-abbauender Enzyme (RNasen) vorhanden sind [20] und erwartungsgemäß einen sofortigen Abbau von freien mRNA-Molekülen bewirken sollten. Es wird allerdings vermutet, dass zirkulierende RNA vor dem Angriff von Serum-RNasen durch assoziierte Moleküle (zum Beispiel als RNA-Proteolipidkomplexe) geschützt sind [20]. Eigene Untersuchungen deuten speziell darauf hin, dass extrazelluläre RNA im Serum innerhalb von Apoptosekörperchen vorliegen könnte und so vor einem Angriff RNA-abbauender Enzyme geschützt wird [21]. Als Ursprungsquelle tumorspezifischer zirkulierender mRNA im Serum/Plasma kommen der Zerfall von nekrotischen Tumorzellen in RNA-enthaltende Vesikel [22] und eine aktive Sekretion von RNA-Molekülen durch Tumorzellen [23] in Betracht. Da bisher nur sehr wenige Daten zum Nachweis zirkulierender mRNA bei Tumorpatienten vorliegen, ist die prognostische Bedeutung dieser Analysetechnik derzeit unklar. Untersuchungen zur Expression extrazellulärer mRNA bei Patienten mit Mammakarzinom konnten bestätigen, dass in einem Teil der Fälle der Tumormarker im Plasma, nicht aber in den parallel analysierten Vollblutproben nachweisbar ist [24]. Weiterhin gelang der Nachweis extrazellulärer tumorassoziierter mRNA teilweise bereits in sehr frühen Tumorstadien [24]. Die vorliegenden Studien zum Nachweis freier RNA bei Tumorpatienten geben Anlass zur Hoffnung, dass neue diagnostische Ansätze entwickelt werden können, deren zukünftige Anwendung in der Frühdiagnostik, Therapieeffizienzkontrolle und im Patientenmonitoring nach erfolgter Therapie liegen. Weitere Forschungsanstrengungen zur Etablierung und Optimierung der Methodik und zur Standardisierung der Technik sind erforderlich, um den klinischen Wert dieses neuen diagnostischen Ansatzes sicher beurteilen zu können.

\section{Literatur}

${ }^{1}$ Garbe C, Schaumburg-Lever G. Klinik und Histologie des malignen Melanoms. In: Garbe C, Dummer R, Kaufmann R, Tilgen W (Hrsg). Dermatologische Onkologie. Berlin Heidelberg New York Tokyo: Springer, 1997

${ }^{2}$ Heimdal K, Hannisdal E, Gundersen S. Regression analysis of prognostic factors in metastatic malignant melanoma. Eur J Cancer Clin Oncol 1989; 25: 1219-1223

${ }^{3}$ Henze G, Dummer R, Joller-Jemelka H, Böni R, Burg G. Serum S100, a marker for disease monitoring in metastatic melanoma. Dermatology 1997; 194: $208-212$

${ }^{4}$ Pelkey TJ, Frierson HF, Bruns DE. Molecular and immunological detection of circulating tumor cells and micrometastasis from solid tumors. Clin Chem 1996; 42: 1369-1381
${ }^{5}$ Miettinen M. Keratin subsets in spindle cell sarcomas. Keratins are widespread but synovial sarcoma contains a distinctive keratin polypeptide pattern and desmoplakins. Am J Pathol 1991; 138: 505 - 513

${ }^{6}$ Thomas P, Battifora H. Keratins versus epithelial membrane antigen in tumor diagnosis- an immunohistochemical comparison of five monoclonal antibodies. Hum Pathol 1987; 18: 728 - 734

${ }^{7}$ Smith B, Selby P, Southgate J, Pittman K, Bradley C, Blair GE. Detection of melanoma cells in peripheral blood by means of reverse transcriptase and polymerase chain reaction. Lancet 1991; 338: 1227-1229

${ }^{8}$ Brossart P, Keilholz U, Willhauck M, Scheibenbogen C, Mohler T, Hunstein W. Hematogenous spread of malignant melanoma cells in different stages of disease. J Invest Dermatol 1993; 101: 887-889

${ }^{9}$ Seiter S, Rappl G, Tilgen W, Ugurel S, Reinhold U. Facts and pitfalls in the detection of tyrosinase mRNA in the blood of melanoma patients by RT-PCR. In: Reinhold U, Tilgen W (Hrsg). Minimal residual disease in melanoma. Recent Results in Cancer Res. Vol. 158. Berlin Heidelberg New York Tokyo: Springer, 2000

${ }^{10}$ Reinhold U, Berkin C, Bosserhoff AK, Deutschmann A, Garbe C, Gläser R, Hein R, Krahn G, Peter RU, Rappl G, Schittek B, Seiter S, Ugurel S, Volkenandt $M$, Tilgen $W$. Interlaboratory evaluation of a new reverse transcriptase polmerase chain reaction-based enzyme-linked immunosorbent assay for the detection of circulating tumor cells: A multicenter study of the dermatologic cooperative oncology group. J Clin Oncol 2001; 19: 1723-1727

${ }^{11}$ Marincola FM, Jaffeee EM, Hicklin DJ, Ferrone S. Escape of solid human tumors from T-cell recognition: molecular mechanisms and functional significance. Adv Immunol 2000; 74: 181 - 273

${ }^{12}$ Hoon DSB, Wang Y, Dale PS, Conrad AJ, Schmid P, Garrison D, Kuo C, Foshag LJ, Nizze AJ, Morton DL. Detection of occult melanoma cells in blood with a multiple-marker polymerase chain reaction assay. J Clin Oncol 1995; 13: 2109-2116

${ }^{13}$ Sarantou T, Chi DD, Garrison DA, Conrad AJ, Schmid P, Morton DL, Hoon DSB. Melanoma-associated antigens as messenger RNA detection markers for melanoma. Cancer Res. 1997; 57: 1371 - 1376

14 Taback B, Morton DL, O’Day SJ, Nguyen DH, Nakayama T, Hoon DSB. The clinical utility of multimarker RT-PCR in the detection of occult metastasis in patients with melanoma. In: Reinhold $\mathrm{U}$, Tilgen $\mathrm{W}$ (Hrsg). Minimal residual disease in melanoma. Recent Results in Cancer Res. Vol. 158. Berlin Heidelberg New York Tokyo: Springer, 2000

${ }^{15}$ de Vries TJ, Fourkour A, Punt CJ, van de Locht LT, Wobbes T, van den Bosch S, de Rooij MJ, Mensink EJ, Ruiter DJ, van Muijen GN. Reproducibility of detection of tyrosinase and MART-1 transcripts in the peripheral blood of melanoma patients: a quality control study using realtime quantitative RT-PCR. Br J Cancer 1999; 80: 883-891

${ }^{16}$ Max N, Wolf K, Spike E, Thiel E, Keilholz U. Nested quantitative real time PCR for detection of occult tumor cells. In: Reinhold U, Tilgen W (Hrsg). Minimal residual disease in melanoma. Recent Results in Cancer Res. Vol. 158. Berlin Heidelberg New York Tokyo: Springer, 2000

17 Kopreski MS, Benko FA, Kwak LW, Gocke CD. Detection of tumor messenger mRNA in the serum of patients with malignant melanoma. Clin Cancer Res 1999; 5: 1961 - 1965

${ }^{18}$ Hasselmann DO, Rappl G, Rößler M, Ugurel S, Tilgen W, Reinhold U. Detection of tumor-associated circulating mRNA in serum, plasma and blood cell samples from patients with disseminated malignant melanoma. Oncol Rep 2001; 8: 115-118

${ }^{19}$ Reinhold U, Schröder $\mathrm{CH}$. Diagnostische Bedeutung frei zirkulierender Nukleinsäuren. Deutsches Ärzteblatt 2002; 99: A1224-1228

${ }^{20}$ Wieczorek AJ, Rhyner C, Block LH. Isolation and characterization of an RNA-proteolipid complex associated with the malignant state in humans. Proc Natl Acad Sci USA 1985; 82: 3455-3459

${ }^{21}$ Hasselmann DO, Rappl G, Tilgen W, Reinhold U. Extracellular tyrosinase mRNA within apoptotic bodies is protected from degradation in human serum. Clin Chem 2001; 47: 1488-1489

22 Jahr S, Hentze H, Englisch S, Hardt D, Fackelmayer FO, Hesch RD, Knippers R. DNA fragments in the blood of cancer patients: quantitations and evidence for their origin from apoptotic and necrotic cells. Cancer Res 2001; 61: 1659-1665

${ }^{23}$ Carr JM, Dvorak AM, Dvorak HF. Circulating membrane vesicles in leukemic blood. Cancer Res 1985; 45: 5944-5951

${ }^{24}$ Silva JM, Dominguez G, Silva J, Garcia JM, Sanchez A, Rodriguez O, Provencio $M$, Espana $P$, Bonilla F. Detection of epithelial messenger RNA in the plasma of breast cancer patients is associated with poor prognosis tumor characteristics. Clin Cancer Res 2001; 7: 2821 -2825 